\title{
Low-energy anti-kaon nucleon and nucleus interaction
}

\author{
J. Marton ${ }^{1, a}$ \\ Stefan Meyer Institute, Boltzmanngasse 3, 1090 Vienna, Austria
}

\begin{abstract}
Central importance for the physics of strong interaction has the dynamics driven by chiral symmetry breaking in low-energy QCD. Valuable information is provided by high precision x-ray studies of the strong interaction at threshold. In this context precision measurements of the strong interaction induced energy shift and width in kaonic atoms are conducted at LNF/Italy at present and at J-PARC/Japan in the future. New dedicated experimental search for bound systems with anti-kaons are under way at GSI and will be conducted at LNF and J-PARC. These studies will provide urgently needed data for theoretical studies and will open the way to a detailed insight in the low energy regime of the strong force with anti-kaons. Especially interesting are nuclear systems involving strangeness and bound by the strong force, called kaonic nuclear clusters. At present this topic is in intense discussion and the search for these systems is conducted in new experiments (e.g. FOPI at GSI). LEANNIS, an international scientific network in Hadronphysics2 within the European Framework Program FP7, will bring together experimentalists and theorists to perform joint research work on the anti-kaon interaction with nucleons and nuclei. This presentation gives an overview on the open problems and and will present the status and outlook of research activities in this challenging field which is inherently connected with few-body physics.
\end{abstract}

\section{Introduction}

The interaction of negatively charged kaons (anti-kaons, $\bar{K}$ ) with nucleons involves strangeness and is not at all simple nor fully understood. As an example of open issues the sub-threshold s-wave resonance $\Lambda(1405)$ is highlighted: after many decades of research its structure is still an open question and topic of intense research in theory and experiment since it is also a key for the understanding of the strong attraction in $\bar{K} \mathrm{~N}$ below threshold and the behavior of the scattering amplitude at threshold. Presently in some theoretical papers this resonance is discussed as a 2-pole resonance [1] with peaks at $1395 \mathrm{MeV}$ and $1420 \mathrm{MeV}$, respectively, with different couplings to $\pi \Sigma$ and $\bar{K} \mathrm{~N}$ channel. Due to the complicated interaction below threshold a lively discussed question is the existence respectively the properties of kaonic nuclei (also called deeply bound kaonic nuclear clusters) like the prototype system $\mathrm{K}^{-} \mathrm{pp}$, as the simplest case, bound by the strong force.

In the last years the physics of low-energy strong interaction involving anti-kaons experienced a renaissance in theory as well as in experiment. This development is partly due to surprises in experimental findings and experimental and theoretical approaches for solving open questions. Some selected topics of current research are listed below.

- Values of the strong interaction induced shift $\epsilon_{1 s}$ and width $\Gamma_{1 s}$ of the 1s state of kaonic hydrogen and kaonic deuterium: Can the difference between results of former experiments on kaonic hydrogen (KpX [2], DEAR

\footnotetext{
${ }^{\text {a }}$ e-mail: johann.marton@oeaw.ac.at
}

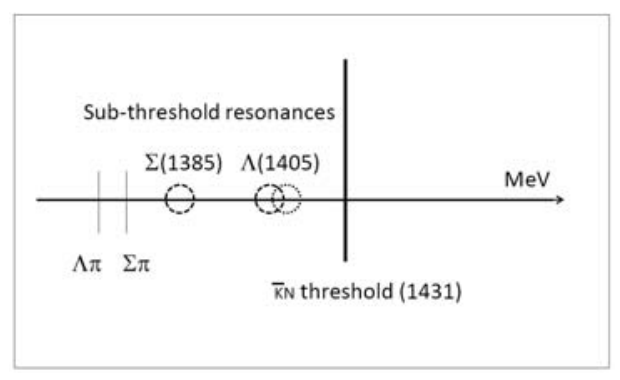

Fig. 1. The strong interaction of anti-kaon with the proton leads to sub-threshold resonances like the $\Sigma(1385)$ in the p wave and the elusive $\Lambda(1405)$ in the s wave, latter being attributed with a 2-pole structure recently.

[3]) be resolved? What accuracy can be obtained with state-of-the-art x-ray spectroscopy?

- What is the best method to extract the anti-kaon-nucleon scattering lengths from the observables $\epsilon_{1 s}$ and width $\Gamma_{1 s}$ ?

- Impact of the threshold data on the chiral symmetry breaking (sigma term) and the strangeness content of the proton.

- How strong is the $\bar{K} \mathrm{~N}$ interaction below threshold?

- What role is $\Lambda(1405)$ playing and what is its nature? Has $\Lambda(1405)$ a 2-pole structure and can this structure be studied in experiments in more detail?

- What is the production mechanism of kaonic nuclei (deeply-bound kaonic nuclear clusters)? What are the binding energies and the widths of possibly formed kaonic nuclei? 
- What is the density of such kaonic nuclei? Is it possible to produce cold dense matter by producing kaonic nuclei with strangeness $\mathrm{S}=-2$ and exploring the QCD phase diagram at these conditions?

The network LEANNIS (Low-Energy Antikaon Nucleon and Nuclear Interaction Studies) [4] within the Integrated Activity HadronPhysics 2 of the $7^{\text {th }}$ European framework program addresses the experimental as well as the theoretical approaches to solve these open questions. This talk is strongly correlated with topics of LEANNIS.

\section{LEANNIS - a liaison between theory and experiment}

LEANNIS is aiming at the understanding of the role of strangeness in the strong force through low-energy antikaon-nucleon and -nucleus interactions. The network will extend the programs at different European research centers like LNF-INFN and GSI. Furthermore, LEANNIS will facilitate the European participation to the upcoming experiments at the J-PARC facility [5] in Japan and the participation of Japanese groups in the European projects.

\subsection{Theory}

The physics of the strong interaction manifests in the dynamics driven by the chiral symmetry breaking of lowenergy QCD. Explicit symmetry breaking by the mass of the strange quark plays an important role when extending the framework to flavour SU(3). Low-energy interactions of anti-kaons with nucleons and nuclei, measured with the highest possible precision, are excellent probes to explore and improve the understanding of this fundamental topic - the entanglement of spontaneous and explicit chiral symmetry breaking. High-precision $\mathrm{K}^{-} \mathrm{p}$ threshold data $\left(\epsilon_{1 s}, \Gamma_{1 s}\right)$ set important constraints for theoretical approaches and have recently been supplemented by the accurate results for kaonic hydrogen from the DEAR experiment [3]. Various theoretical analyses [6,7] point to questions of consistency of the recent DEAR data with previously measured sets of kaon-proton scattering data. It is expected that the follow-up experiment SIDDHARTA at LNF [8] will improve the DEAR data further on and will attempt the very first measurement of $x$-ray transitions in kaonic deuterium. From these quantities the two isospindependent s-wave kaon-nucleon scattering lengths can be extracted in principle separately but theoretical work is necessary to extract these quantities [9]. Accurate results for anti-kaon-nucleon threshold data can be regarded as prerequisite for realistic studies of deeply bound anti-kaonnuclear states. At present, various experimental results and first exploratory theoretical approaches do not yield a conclusive picture about the possible existence and properties of deeply bound states $[10,11]$. A systematic theoretical framework based on chiral effective field theory in combination with non-perturbative methods must be developed to provide a clean theoretical link to the underlying QCD theory. Such a framework will be the basis for unambiguous theoretical studies of kaonic nuclei like $\mathrm{K}^{-} \mathrm{pp}$.

\subsection{Experiment}

In order to solve problems and questions listed in Sect. 1 new experiments were performed at several research centers like GSI/Germany, LNF/Italy recently and dedicated experiments are in preparation at LNF and J-PARC employing new technologies.

\subsubsection{Threshold data - $\mathrm{x}$ ray spectroscopy of kaonic atoms}

Sources of information about the low-energy $\bar{K} \mathrm{~N}$ interaction are (1) scattering data, (2) decay ratios in inelastic reactions, (3) the $\pi \Sigma$ mass spectrum and last but not least (4) x-ray data. The spectroscopy of kaonic atoms provide a rather straightforward access to the low-energy $\mathrm{K} \bar{N}$ interaction near zero energy. Especially interesting are kaonic hydrogen and kaonic deuterium atoms as the simplest atoms with strangeness. The strong interaction in these atoms is affecting measurable only the ground state $(1 \mathrm{~s})$ whereas the strong interaction effect on the higher $\mathrm{n}$ states is negligible. Therefore, from the measured energies of the X-ray transitions to the $1 \mathrm{~s}$ state (K lines) the strong interaction induced shift $\epsilon_{1 s}$ and the width $\Gamma_{1 s}$ can be extracted directly where the relation is given by the following relation

$$
\epsilon_{1 s}=E_{1 s}^{\text {meas }}-E_{1 s}^{\text {calc }}
$$

Here $\mathrm{E}_{1 s}^{\text {meas }}$ is the measured (electromagnetic and strong interaction) and $\mathrm{E}_{1 s}^{\text {calc }}$ (only electromagnetic interaction) the calculated transition energy to the $1 \mathrm{~s}$ state.

This approach implies the knowledge of $\mathrm{E}_{1 s}^{\text {calc }}$ which can be obtained by solving the Klein-Gordon equation and taking correction into account (e.g. vacuum polarization, finite size). Ultimately the accuracy is limited by the uncertainty in the kaon mass. Nevertheless $\mathrm{E}_{1 s}^{\text {calc }}$ can be calculated with an sufficient accuracy of about $1 \mathrm{eV}$ which is much better than the experimental error bar in $\mathrm{E}_{1 s}^{\text {meas }}$.

About a decade ago the sign of $\epsilon_{1 s}$ was found to have a negative value in agreement with the extrapolation of scattering experiments and in disagreement with former experiment. This finding was verified by the DEAR experiment at LNF [3]. However the precision of $\epsilon_{1 s}$ and $\Gamma_{1 s}$ was limited due to the high background in the x-ray spectra which leads to a small signal-to-background ratio and large error bars.

Recently at DA $\Phi N E / L N F$ in Frascati x-ray measurements of kaonic hydrogen, deuterium, ${ }^{3} \mathrm{He}$, and ${ }^{4} \mathrm{He}$ were successfully finished using an array of recently developed large area Silicon Drift Detectors with timing capability (SDDs)[12]. The data taking period starting in 2008 and lasted till autumn 2009. Now the x-ray data are in analysis to extract more precise values of shifts and widths of the $1 \mathrm{~s}$ state (kaonic hydrogen and deuterium) and $2 \mathrm{p}$ state (kaonic helium) as input for theoretical studies. A detailed 
description and preliminary results were presented at this conference [13].

The strong interaction shift of the $2 p$ state in kaonic $\mathrm{He}-4$ was successfully determined in a recent experiment at KEK [14] thus clarifying the previous striking disagreement between the theoretical and experimental values of the $2 p$ shift. Now the value of the $2 p$ state shift turned out to be compatible with $0 \mathrm{eV}$ in agreement with theory. This result was confirmed by the SIDDHARTA experiment [15]. As a next step at J-PARC a new precision experiment on the $\mathrm{X}$-ray spectrum of kaonic helium-3 is in preparation [16]. From the spectroscopy of X-rays feeding the $2 p$ level, the strong-interaction shift and width of the $2 p$ state can be deduced. This information offers a unique possibility to precisely determine the anti-kaon-nucleus strong interaction at vanishing relative energy. Furthermore, from the comparison with recent theory valuable information about kaonic nuclear clusters can be obtained. This experiment at J-PARC (E17) will employ a liquid ${ }^{3} \mathrm{He}$ target surrounded by large area silicon drift detectors for the X-ray detection. $\mathrm{X}$-ray data are essential for the theoretical understanding of the low energy antikaon-nucleon interaction and the basic input for studies of anti-kaonic nuclear bound states.

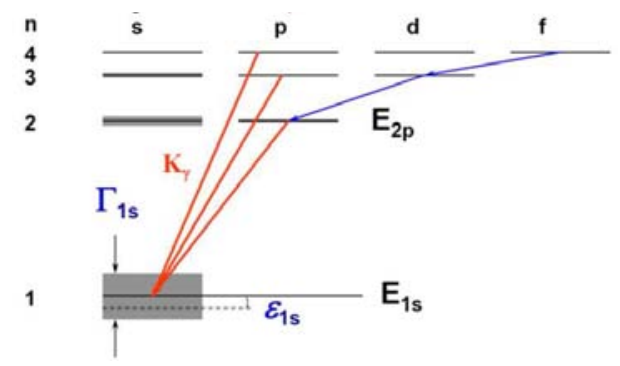

Fig. 2. The strong interaction in kaonic hydrogen and deuterium atoms can be studies by $\mathrm{x}$-ray spectroscopy of the radiative transitions from the $\mathrm{p}$ states to the $1 \mathrm{~s}$ state ( $\mathrm{K} \mathrm{x}$-ray lines).

\subsubsection{Kaonic nuclei}

Reliable and precise results of $\bar{K}$-N threshold data are an essential input for realistic studies of kaonic nuclei. At present, experimental findings and theoretical studies do not yield a consistent picture about the possible existence and properties of deeply bound states. However, results of several experiments employing different methods DISTO (proton collision)[17], FINUDA ( ${ }^{-}$induced reaction)[18] and OBELIX (antiproton-induced reaction) [19])indicate the existence of kaonic nuclei. In this context new data will come from experiments like $\mathrm{pp} 2 \mathrm{~K} \Lambda \mathrm{p}$ [20] which was conducted in 2009 and is in the status of data analysis now.

On the theoretical side the results of phenomenological studies with the Ansatz $\Lambda(1405)=\mathrm{K}^{-} \mathrm{p}$ (AY02) [21], Faddeev calculations (FC)[22-24] and variational calculations (VC) $[25,26]$ on the properties of $\mathrm{K}^{-} \mathrm{pp}$ cover a broad range: The binding energy is ranging from $55-70 \mathrm{MeV}$ (FC) to $20-50 \mathrm{MeV}$ (VC), whereas the decay width covers

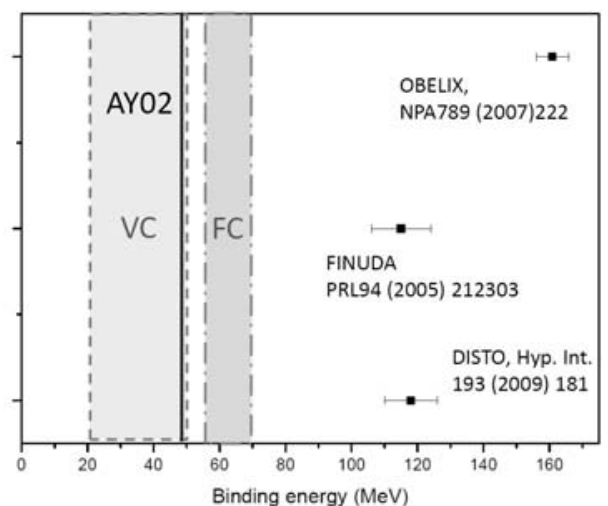

Fig. 3. Binding energy of $\mathrm{K}^{-} \mathrm{pp}$ measured by DISTO [17], FINUDA [18] and OBELIX [19] in comarison with values from theory.

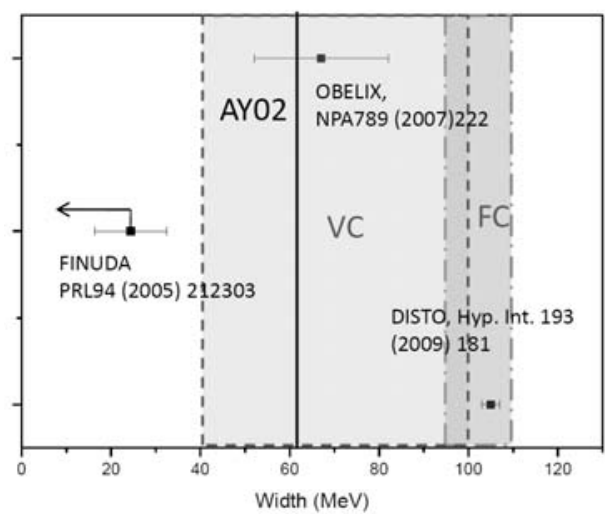

Fig. 4. Decay width of $\mathrm{K}^{-} \mathrm{pp}$ measured by DISTO [17], FINUDA [18] and OBELIX [19] in comarison with values from theory.

95-110 MeV (FC) to 40-100 MeV (VC). Therefore, theoretical studies on kaonic nuclei have to be further improved but need the input from experiments.

New dedicated experiments like AMADEUS [27] and E15 [28] at J-PARC are in preparation .

AMADEUS is aiming at exclusive studies of the production and decay of kaonic nuclei produced by stopped antikaon absorption at the DA $\Phi$ NE $\Phi$-factory at LNF in Frascati. The AMADEUS apparatus will provide the detection of all charged, and neutral outgoing particles including photons using the $4 \pi$ detector system of KLOE, cryogenic targets and a kaon tagging system similar to SIDDHARTA. The detector systems will employ new technologies like GEM based detectors [29] and photo-detection with silicon photomultipliers (SiPMs)[30]. The goals will be, besides high statistics studies of various absorption processes of stopped anti-kaons in light nuclei, the possible unambiguous detection of various light anti-kaonic nuclear systems, such as $\mathrm{K}^{-} \mathrm{pp}, \mathrm{K}^{-} \mathrm{ppn}$, and $\mathrm{K}^{-} \mathrm{pnn}$ with data on the binding energies, total widths and all partial decay widths. From Dalitz plots of 3-body decays of kaonic nuclei detailed structure information (such as parity, angular momentum, radii and densities) can be deduced. Such data would give valuable input in theories for the description of dense, cold, strongly interacting systems. 
The E15 experiments will use the in-flight reaction of antikaons produced at J-PARC to produce kaonic nuclei.

New data on heavy ion induced reactions were taken in 2009 with the FOPI apparatus at GSI. Possibly formed multi-baryonic states are reconstructed in channels that contain charged particles only, like $\mathrm{ppnK}^{-} \rightarrow \Lambda \mathrm{d}$. The analyses aim at the reconstruction of $\Lambda$ hyperons and analysis of the correlation with other emitted particles. The interpretation of the data will clearly benefit from the close interaction with theoreticians participating in the LEANNIS network.

\section{Summary}

The recent history of research in low-energy anti-kaon interaction physics is characterized by some puzzles (kaonic hydrogen, kaonic helium) which were solved in the last years - but still challenging problems remain and are subject to new experiments and theoretical work. The LEANNIS network was set up to comprise in an interactive manner relevant projects in strangeness physics which deal with low energy interactions of anti-kaons with nucleons and nuclei including the the search for the still enigmatic deeply bound anti-kaonic nuclei. The activities are concentrated on developing new strategies, both in experimental and theoretical sectors, to attack the numerous problems in the field. The development of new experimental methods and techniques, e.g. SDDs as high-performance X-ray detectors, GEM based detectors and new SiPM photon detectors. Major European institutes working in this field are participating to this network, therefore a platform is given to strengthen and bundle the efforts. A close collaboration of experimentalists with theoreticians, for the analysis of the new upcoming experimental results and their interpretation in the framework of the non perturbative QCD with strangeness is an essential goal of this network in order to further develop this research field and gain a detailled understanding of the many facets of low-energy anti-kaon nucleon and -nucleus interactions.

\section{References}

1. W. Weise and R. Härtle, Nucl. Phys. A804 (2008) 173.

2. M. Iwasaki et al., Phys. Rev. Lett. 78 (1997) 3067.

3. G. Beer et al., Phys. Rev. Lett. 94 (2005) 212302.

4. http://www.oeaw.ac.at/smi/index.php?id=83

5. http://j-parc.jp/index-e.html

6. J.A. Oller, J. Prades and M. Verbeni, Phys. Rev. Lett. 95 (2005) 172502.

7. B. Borasoy, R. Nissler, and W. Weise, Phys. Rev. Lett. 94 (2005)213401; Eur. Phys. J. A25 (2005) 79.

8. http://www.lnf.infn.it/

9. M. Lage, U.-G. Meißner and A. Rusetzky, Hyp. Int. 193 (2009) 69.

10. E. Friedman and A. Gal, Physics Reports 452 (2007) 89.

11. J. Zmeskal, Progress in Particle and Nuclear Physics 61 (2008) 512.
12. J. Marton et al., Proc. of the $8^{\text {th }}$ Symposium on Radiation Maesurement and Applications, Berkely, 2008, in print.

13. S. Okada et al., this Conference.

14. S. Okada et al., Physics Letters B653 (2007) 387.

15. M. Bazzi et al., Physics Letters B681 (2009) 310.

16. M. Iio, this Conference.

17. T. Yamazaki et al.,Hyp. Int. 193 (2009) 181.

18. M. Agnello et al., Phys. Rev. Lett. 94 (2005) 212303.

19. T. Bressani, et al.Hyperfine Interact. 193 (2009) 201.

20. K. Suzuki et al., Nucl.Phys.A827 (2009) 312C.

21. T. Yamazaki, Y. Akaishi Physics Letters $B 535$ (2002) 70, Proceedings of the Japan Academy, Ser.B 82 (2007).

22. N. Shevchenko et al. PRL 98 (2007) 082301.

23. N. Shevchenko et al. PRC 76(2007) 044004.

24. Y. Ikeda, T. Sato PRC 76 (2007) 035203.

25. A. Dote, T. Hyodo, W. Weise NPA 804 (2008) 197.

26. A. Dote, T. Hyodo, W. Weise PRC 79 (2009) 014003.

27. O. Vázquez Doce, Hyperfine Interact. 193 (2009) 195.

28. T. Tsukuda, this Conference.

29. M.P. Lener et al., Nucl. Instr. Meth. in press.

30. K. Suzuki et al., Nucl. Instr. Math. A620 (2009) 75. 